3 Hot A, Maunoury C, Poiree S, et al. Diagnostic contribution of positron emission tomography with [18F] fluorodeoxyglucose for invasive fungal infections. Clin Microbiol Infect 2011; 17: 409-417.

4 Veronesi G, Travaini LL, Maisonneuve P, et al. Positron emission tomography in the diagnostic work-up of screening-detected lung nodules. Eur Respir J 2015; 45: 501-510.

\title{
Amoxicillin for clinically unsuspected pneumonia in primary care: subgroup analysis
}

\author{
To the Editor:
}

Antibiotic treatment is recommended for pneumonia [1, 2]. However, some studies have suggested that the milder spectrum of pneumonia in primary care does not have important adverse consequences if missed and, therefore, managed without antibiotics [3-5]. Better insight in the effects of antibiotics for pneumonia in primary care could improve patient information and management.

The aim of our study was to assess whether antibiotic treatment influenced outcome in patients presenting with acute lower respiratory tract infection (LRTI) with a radiologically proven, but clinically unsuspected, pneumonia compared to those without pneumonia.

This was a secondary analysis of a randomized, placebo controlled trial of amoxicillin for acute LRTI in 16 primary research networks in 12 European countries from October 2007 until April 2010. More details on this GRACE-10 study (Genomics to combat Resistance against Antibiotics in Community-acquired LRTI in Europe, www.gracelrti.org) have been reported elsewhere [6]. Eligible patients were aged $\geqslant 18$ years who consulted their general practitioner (GP) for the first time with an acute cough (duration of $\leqslant 28$ days) as the main symptom. Exclusion criteria were clinically suspected pneumonia [7], based on focal chest signs (focal crepitations and bronchial breathing) and systemic features (high fever, vomiting and severe diarrhoea); pregnancy, allergy to penicillin, treatment with antibiotics in the previous month and immunodeficiency. The study was approved by ethics committees in all participating countries and all participants provided written informed consent.

Patients who agreed to randomisation were allocated to receive amoxicillin $(1 \mathrm{~g}$ three times daily for 7 days) or placebo, by the GP dispensing sequentially numbered randomised containers.

GPs recorded patients' clinical signs, and comorbidities on a case report form. They also registered 14 baseline symptoms (cough, phlegm, shortness of breath, wheeze, runny nose, fever, chest pain, muscle aching, headache, disturbed sleep, feeling generally unwell, interference with normal activities/work, confusion/ disorientation and diarrhoea) on a 4-point Likert-scale from "no problem" to "severe problem". Baseline symptom severity was calculated by summing the scores of the symptoms and rescaling them to make them range between 0 and 100. Patients filled in a daily symptom diary during their illness for up to 28 days for the same symptoms on a 7 -point Likert scale $(0=$ no problem, $1=$ very little problem, $2=$ slight problem, $3=$ moderately bad, $4=$ bad, $5=$ very bad and $6=$ as bad as it could be). This diary was previously validated and shown sensitive to change [8]. If the diary was not returned after 4 weeks, brief information about symptom duration and severity were collected with either a short questionnaire or a standardised telephone call.

Chest radiographs were taken for each patient within 7 days of first presentation, but preferably within 3 days. Pneumonia was determined by radiologists, who were blind to all other information when they judged chest radiographs. In analysis reported here we defined pneumonia as all patients in whom the radiologist diagnosed pneumonia based on the chest radiograph [9].

Outcomes were duration of symptoms rated by patients as "moderately bad or worse" after initial presentation, symptom severity on days 2-4 after the index consultation, and worsening of illness, defined as a revisit to the GP with worsening symptoms, new symptoms, new signs, or illness necessitating admission to hospital within 4 weeks after the first consultation [6].

The effectiveness of the antibiotics was compared in patients with radiologically proven pneumonia compared to those without pneumonia for all three outcomes. Data were analysed using linear regression models. Cox regression was used for the duration of symptoms allowing for censoring, simple linear regression for 
symptom severity and logistic regression for worsening of illness. Interaction terms were used to estimate differences in effectiveness of antibiotics in those with radiologically proven pneumonia compared to those without pneumonia for all three outcomes. To assess whether the effect of antibiotics in patients with radiologically proven pneumonia was modified by illness duration before index consultation and baseline symptom severity, we used interaction terms for each outcome. Number needed to harm was calculated.

A total of 2055 patients with acute cough were recruited in the randomised controlled trial (RCT), of which 1905 (93\%) underwent a chest radiograph. Patients with a chest radiograph of insufficient quality $(n=20)$ were excluded, resulting in 1885 subjects for analysis. Patients without chest radiograph results were younger and had a higher baseline symptom severity. The mean \pm sD age of the 1885 subjects was $49 \pm 16$ years and $41 \%(n=769)$ were male. Radiologically proven pneumonia was present in $56(3 \%)$ out of 1885. Baseline characteristics did not differ significantly between those with and without pneumonia nor in patients with pneumonia between those allocated to amoxicillin $(n=23)$ and to those allocated to placebo $(n=33)$.

Patients with radiologically proven pneumonia who were allocated to antibiotics had faster resolution of symptoms rated "moderately bad or worse" than those allocated to placebo (median 5 days versus 11 days, HR 2.20, 95\% CI 1.18-4.08, $\mathrm{p}=0.013$; interaction term for the difference in effect between those with and without pneumonia 2.04, $\mathrm{p}=0.024)$ and lower mean $(95 \% \mathrm{CI})$ symptom severity on days $2-4$ were -0.68 $(-1.28-0.08)(\mathrm{p}=0.027)$, although the interaction term was of borderline significance (interaction term -0.63 ; $\mathrm{p}=0.050$ ) (table 1). Development of new or worsening symptoms was not different in those allocated to antibiotics as compared to those allocated to placebo (OR $0.33,95 \%$ CI $0.10-1.11 ; \mathrm{p}=0.074$ ) and the effect of antibiotics between those with and without pneumonia was not different (interaction term 0.38; $\mathrm{p}=0.125$ ). In patients with radiologically proven pneumonia the effect of antibiotics was not modified by illness duration before index consultation and baseline symptom severity for all three outcomes (results not shown) In patients without pneumonia amoxicillin showed no effect (table 1). Nausea, rash, or diarrhoea was recorded by 239 (29\%) out of 822 patients in the amoxicillin group and $196(24 \%)$ out of 811 in the placebo group (number needed to harm 21, 95\% CI 11-159; p=0.025). Two patients in the placebo group and one in the amoxicillin group needed to be admitted to hospital. No study-related deaths were noted.

Our findings are consistent with recommendations in current guidelines $[1,2]$ that those with radiologically proven pneumonia appear to derive beneficial effect from antibiotics on symptom duration and symptom severity. The findings from our placebo-controlled study will compliment trials of head to head comparisons between antibiotic classes summarised in the Cochrane review of antibiotics for pneumonia in primary care [10].

In this analysis only patients with clinically unsuspected pneumonia were studied that represent likely the somewhat milder end of the clinical spectrum. In these patients we saw a clear and relevant effect of antibiotics. In addition, power calculation of the original RCT did not include this subgroup analysis of patients with pneumonia, which is another reason why we could have missed some true effects of the intervention. Finally, stratifying all trial patients according to the presence or absence of radiologically proven pneumonia makes it uncertain whether confounders were still randomly distributed between these

\begin{tabular}{|c|c|c|c|c|c|c|c|}
\hline & Amoxicillin & Placebo & Interaction term $(95 \% \mathrm{CI})^{\#}$ & p-value & Analysis & p-value & Missing $\mathrm{n}(\%)$ \\
\hline \multicolumn{8}{|c|}{$\begin{array}{l}\text { Time to resolution of symptoms } \\
\text { rated moderately bad days }\end{array}$} \\
\hline Pneumonia present & $5(2-9)$ & $11(7-20)$ & $2.04(1.10-3.80)$ & 0.024 & $2.20(1.18-4.08)^{\pi}$ & 0.013 & 8 (14.3) \\
\hline Pneumonia absent & $6(3-12)$ & $7(4-13)$ & & & $1.05(0.95-1.16)^{9}$ & 0.363 & $182(9.7)$ \\
\hline Pneumonia present & $1.48 \pm 0.95$ & $2.16 \pm 1.02$ & $-0.63(-1.27-0.00)$ & 0.050 & $-0.68(-1.28--0.08)^{+}$ & 0.027 & 10 (17.9) \\
\hline Pneumonia absent & $1.82 \pm 1.05$ & $1.87 \pm 1.08$ & & & $-0.05(-0.15-0.06)^{+}$ & 0.365 & 232 (12.3) \\
\hline \multicolumn{8}{|l|}{ Worsening of illness } \\
\hline Pneumonia present & $5 / 23(22)$ & 15/33 (46) & $0.38(0.11-1.30)$ & 0.125 & $0.33(0.10-1.11)^{\S}$ & 0.074 & $0(0.0)$ \\
\hline Pneumonia absent & 149/934 (16) & $160 / 895(18)$ & & & $0.87(0.68-1.11)^{\S}$ & 0.272 & $0(0.0)$ \\
\hline
\end{tabular}

Data are presented as median (interquartile range, mean $\pm \mathrm{SD}$, or $\mathrm{n} / \mathrm{N}(\%)$, unless otherwise stated. \#: interaction addresses the comparison of the effects of amoxicillin between those with and without pneumonia; ": data presented as hazard ratio (95\% Cl); ${ }^{+}$: data presented as difference $(95 \% \mathrm{Cl}) ;{ }^{\S}$ : data presented as odds ratio $(95 \% \mathrm{CI})$. 
two groups. However, baseline characteristics did not differ significantly between those allocated to amoxicillin and those allocated to placebo in this subgroup.

Radiologically proven pneumonia which is not clinically suspected at presentation does benefit from antibiotic treatment in terms of symptom duration and symptom severity. This suggests that efforts to detect even the milder and less clear cases of pneumonia in primary care are worthwhile. Since it is not feasible or cost-effective to perform a chest radiograph in all patients with signs and symptoms of a LRTI in primary care, diagnostic models and the use of point-of-care tests could be useful in improving diagnostic strategies in these patients.

@ERSpublications

Clinically unsuspected pneumonia does benefit from antibiotic treatment in terms of duration and severity of symptoms http://ow.ly/T46up

Jolien Teepe ${ }^{1}$, Paul Little ${ }^{2}$, Nori Elshof ${ }^{1}$, Berna D.L. Broekhuizen ${ }^{1}$, Michael Moore ${ }^{2}$, Beth Stuart ${ }^{2}$, Chris C. Butler ${ }^{3,4}$, Kerenza Hood ${ }^{5}$, Margareta Ieven ${ }^{6}$, Samuel Coenen ${ }^{6,7}$, Herman Goossens ${ }^{6}$ and Theo J.M. Verheij ${ }^{1}$ on behalf of the GRACE consortium

${ }^{1}$ University Medical Center Utrecht, Julius Center for Health Sciences and Primary Care, Utrecht, The Netherlands. ${ }^{2}$ University of Southampton Medical School, Primary Care Medical Group, Southampton, UK. ${ }^{3}$ Nuffield Dept of Primary Care Health Sciences, Oxford University, Oxford, UK. ${ }^{4}$ Cardiff University School of Medicine, Institute of Primary Care and Public Health, Cardiff, Wales. ${ }^{5}$ Cardiff University, Institute for Translation, Innovation, Methodology and Engagement, Cardiff, Wales. ${ }^{6}$ University of Antwerp, Laboratory of Medical Microbiology, Vaccine and Infectious Diseases Institute (VAXINFECTIO), Antwerp, Belgium. ${ }^{7}$ University of Antwerp, Centre for General Practice, Primary and Interdisciplinary Care (ELIZA), Antwerp, Belgium.

Correspondence: Berna D.L. Broekhuizen, University Medical Center Utrecht, Julius Center for Health Sciences and Primary Care, Stratenum 6.131, PO Box 85500, 3508 GA Utrecht, The Netherlands. E-mail: b.d.l.broekhuizen@umcutrecht.nl

Received: Feb 102015 | Accepted after revision: Aug 29 2015 | First published online: Nov 52015

Support statement: This study was part of the GRACE project (www.grace-lrti.org), funded by the 6th Framework Program of the European Commission (Reference: LSHM-CT-2005-518226). The GRACE project has been financially supported through the European Science Foundation (ESF), in the framework of the Research Networking Programme TRACE (www.esf.org.trace) and by the Research Foundation Flanders (FWO; Belgium). The current analysis was supported by the SBOH (www.sboh.nl), employer of Dutch general practitioner trainees. The funding sources were not involved in the design, conduct, analysis and interpretation of the data, or in the writing of the paper. Funding for this article has been deposited with FundRef.

Clinical trials: this study is registered at https://eudract.ema.europa.eu/ with identifier number 2007-001586-15; http://public. ukcrn.org.uk/search/ with identifier number ID 4175; http://www.isrctn.com/login with identifier number 52261229; and http://www.fwo.be/ with identifier number G.0274.08N.

Conflict of interest: Disclosures can be found alongside the online version of this article at erj.ersjournals.com

Acknowledgements: Contributors: Chris Butler, Samuel Coenen, Herman Goossens, Kerenza Hood, Paul Little, and Theo Verheij conceived the study idea and designed the study. Jolien Teepe, Nori Elshof, Lidewij Broekhuizen and Beth Stuart interpreted data and performed the analyses. Jolien Teepe, Nori Elshof, Lidewij Broekhuizen, and Theo Verheij wrote a first draft of the manuscript, Paul Little revised the first draft critically and thoroughly, and all mentioned co-authors critically revised the manuscript. The guarantor is Theo Verheij.

The clinical part of the GRACE consortium consists of: Jolien Teepe (Netherlands), Lidewij Broekhuizen (Netherlands), Curt Brugman (Netherlands), Chris Butler (UK), Kerenza Hood (UK), Samuel Coenen (Belgium), Herman Goossens (Belgium), Margareta Ieven (Belgium), Christine Lammens (Belgium), Jordi Almirall (Spain), Francesco Blasi (Italy), Slawomir Chlabicz (Poland), Mel Davies (UK), Maciek Godycki-Cwirko (Poland), Helena Hupkova (Slovakia), Janko Kersnik (Slovenia), Artur Mierzecki (Poland), Sigvard Mölstad (Sweden), Michael Moore (UK), Beth Stuart (UK), Gilly O’Reilly (UK), Mark Mullee (UK), Tom Schaberg (Germany), An De Sutter (Belgium), Antoni Torres (Spain), Pia Touboul (France), Paul Little (UK), and Theo J.M. Verheij (Netherlands).

We would like to thank the entire GRACE team for their diligence, expertise and enthusiasm. We are indebted to all of the patients who consented to be part of GRACE, without whom this study would not have been possible.

\section{References}

1 Woodhead M, Blasi F, Ewig S, et al. Guidelines for the management of adult lower respiratory tract infections full version. Clin Microbiol Infect 2011; 17: Suppl. 6, E1-E59.

2 National Institute for Health and Care Excellence. Pneumonia: Diagnosis and management of community- and hospital-acquired pneumonia in adults. Date last updated: December, 2014. Date last accessed: February, 2015.

3 Macfarlane J, Holmes W, Gard P, et al. Prospective study of the incidence, aetiology and outcome of adult lower respiratory tract illness in the community. Thorax 2001; 56: 109-114.

4 Macfarlane JT, Colville A, Guion A, et al. Prospective study of aetiology and outcome of adult lower-respiratory-tract infections in the community. Lancet 1993; 341: 511-514.

5 Hopstaken RM, Coenen S, Butler CC. Treating patients not diagnoses: challenging assumptions underlying the investigation and management of LRTI in general practice. J Antimicrob Chemother 2005; 56: 941-943. 
Little P, Stuart B, Moore M, et al. Amoxicillin for acute lower-respiratory-tract infection in primary care when pneumonia is not suspected: a 12-country, randomised, placebo-controlled trial. Lancet Infect Dis 2013; 13: 123-129.

7 British Thoracic Society. BTS guidelines for the management of community acquired pneumonia in adults. Thorax 2001; 56: Suppl. 4, iv1-iv64.

8 Watson L, Little P, Moore M, et al. Validation study of a diary for use in acute lower respiratory tract infection. Fam Pract 2001; 18: 553-554.

9 van Vugt SF, Broekhuizen BD, Lammens C, et al. Use of serum C reactive protein and procalcitonin concentrations in addition to symptoms and signs to predict pneumonia in patients presenting to primary care with acute cough: diagnostic study. BMJ 2013; 346: f2450.

10 Pakhale S, Mulpuru S, Verheij TJM, et al. Antibiotics for community-acquired pneumonia in adult outpatients. Cochrane Database Syst Rev 2014; 10: CD002109.

\section{Tuberculosis screening using ability to provide sputum in an endemic emergency department}

To the Editor:

Tuberculosis causes an enormous burden of morbidity and mortality worldwide [1]. Increasing rates of multidrug-resistant (MDR) and extensively drug-resistant (XDR) tuberculosis pose a serious threat to ongoing efforts in controlling the epidemic, particularly in low- and middle-income countries [2].

The World Health Organization (WHO) recommends tuberculosis screening via sputum smear examination in adults attending outpatient health facilities who report cough for $\geqslant 2$ weeks [3]. Little is known about optimal case finding definitions in emergency departments where the prevalence of tuberculosis appears to be higher than outpatient clinics [4]. Furthermore, emergency departments are highly important congregate settings bringing together the sickest diagnosed and undiagnosed tuberculosis patients with a vulnerable population, and may be the main portal of entry to care for persons who otherwise would not seek routine medical attention in outpatient clinics.

We assessed the use of a simple case finding strategy based on obtaining a sputum sample for acid-fast smear and culture in all emergency department comers who could produce a sputum sample, regardless of reason for emergency department visit, report of cough symptoms or cough duration.

The study was conducted between July 2005 and August 2006 in Hospital Nacional Dos de Mayo, a large public hospital in Lima, Peru, serving a low-income area with a tuberculosis rate of 113 cases per 100000 inhabitants [5]. Patients evaluated in the emergency department were invited to participate in the study if they met the following criteria: $\geqslant 18$ years of age, $\geqslant 2 \mathrm{~h}$ stay in the emergency department, and ability to provide an adequate sputum sample for acid-fast bacilli (AFB) smear and culture. Patients who were receiving tuberculosis treatment at enrollment were excluded. Patients were enrolled from 08:00 h to 20:00 h on Monday to Saturday. Participants provided written informed consent and were administered a standardised questionnaire to collect sociodemographic and clinical data including age, gender, residence location, occupation, medical history, tuberculosis risk factors and symptoms. One sputum sample was collected per patient.

Ziehl Neelsen AFB smear microscopy was performed on all sputum samples at the hospital laboratory. Sputum samples were cultured on acid-buffered Ogawa media for 8 weeks [6]. Susceptibility testing was performed at the Lima-Ciudad Regional Laboratory using the proportional method of CANETTI and GROSSET [7].

Pulmonary tuberculosis (PTB) was defined as having a sputum culture positive for Mycobacterium tuberculosis. MDR-tuberculosis was defined as cultures resistant to at least isoniazid and rifampin. PTB patients were offered counselling for HIV testing. Stata software (version 12.0; StataCorp, Texas) was used for analysis and a p-value $\leqslant 0.05$ was considered significant. The study was approved by the hospital ethics committee.

Over the study period, 931 patients were enrolled. Of these, 174 (18.7\%) had PTB. Table 1 shows the characteristics of PTB patients by cough report. $42(24.1 \%)$ of the 174 PTB patients reported no cough. 26 (14.9\%) PTB patients had cough for <2 weeks. The remaining 106 (60.9\%) PTB patients reported 